\title{
Knowledge of playing-related musculoskeletal disorders among classical piano students at tertiary institutions in Malaysia
}

\begin{abstract}
Performance injuries among musicians have been widely discussed for decades. However, despite the growing number of classical pianists, this is still a new issue in Malaysia. Therefore, this study aimed to investigate the level of knowledge of playing-related musculoskeletal disorders (PRMDs) among tertiary music students in Malaysia. A survey was conducted among classical piano students at tertiary institutions of Kuala Lumpur and Selangor. Out of 192 respondents, 76\% knew that piano playing can cause PRMDs. Slightly over three-quarters of respondents $(77.1 \%)$ learned about PRMDs from music educators. The survey revealed that the belief in 'no pain, no gain' was still ingrained in their minds, as $50.5 \%$ respondents believed that pain experienced while playing the piano was normal and $51.6 \%$ of them considered that pain must be experienced to improve their piano skill. The respondents were also scored on questions on terminologies of pianist injury and specific PRMD examples: $7.8 \%$ of respondents scored high in the questions on the general terminology, while $99.5 \%$ of them scored low in the questions on the specific examples of PRMDs. This finding indicated a lack of knowledge of specific musicians' injuries among classical piano students. The attitudes to pain and the level of understanding of the significance of potential injuries indicate that increasing PRMD awareness and introducing courses on PRMD prevention at tertiary institutions are warranted.
\end{abstract}

Keyword: Knowledge of playing; Musculoskeletal disorders; Classical piano students; Tertiary institutions; Malaysia 